\title{
Health care utilization and costs associated with switching from DPP-4i to GLP-1RA or SGLT2i: an observational cohort study
}

\author{
Terri Victoria Newman, PharmD; Kiraat D Munshi, PhD; Lynn M Neilson, PhD; Chester B Good, MD, MPH; \\ Elizabeth C S Swart, BS; Yan Huang, MS; Rochelle Henderson, PhD; and Natasha Parekh, MD, MS
}

\section{What is already known about this subject}

- Recent guidelines from the American Diabetes Association and the American Association of Clinical Endocrinologists/American College of Endocrinology recommend the use of glucagon-like peptide 1 receptor agonists (GLP-1RA) and sodiumglucose cotransporter 2 inhibitors (SGLT2i) over dipeptidyl peptidase-4 inhibitors (DPP-4i) as second-line agents for those with or without cardiovascular disease.

- DPP-4i have higher utilization rates compared with GLP-IRA and SGLT2i, despite recent clinical recommendations and the cardiovascular and mortality benefit seen with these newer agents.

- It remains unclear how switching to SGLT2i or GLP-1RA from DPP-4i affects health care utilization and costs in the real-world setting.

\section{What this study adds}

- This study provides real-world evidence on the effect of switching from DPP-4i to GLP-1RA and SGLT2i agents on health care utilization and cost outcomes.

- Results demonstrated that switching to an SGLT2i or GLP-1RA was associated with fewer inpatient hospitalizations and higher total pharmacy costs for GLP-1RA.

- This study carries important implications for payers, providers, and policymakers to consider the extent to which higher pharmacy costs may offset medical savings associated with the use of SGLT2i or GLP1-RA.

\section{Author affiliations}

Terri Victoria Newman, PharmD, Department of Pharmacy and Therapeutics, University of Pittsburgh School of Pharmacy, Pittsburgh, PA. Kiraat D Munshi, PhD, and Rochelle Henderson PhD, Express Scripts, St. Louis, MO. Lynn M Neilson, PhD; Elizabeth C S Swart, BS; and Yan Huang, MS, Center for Value-Based Pharmacy Initiatives and HighValue Care, UPMC Health Plan Insurance Services Division, Pittsburgh, PA. Chester B Good, MD, MPH, Center for Value-Based Pharmacy Initiatives and High-Value Care, UPMC Health Plan Insurance Services Division, Pittsburgh, PA, and University of Pittsburgh Division of General Internal Medicine, Pittsburgh, PA. Natasha Parekh, MD, MS, The Queen's Health System, Honolulu, HI, and University of Hawaii School of Medicine, Honolulu.

AUTHOR CORRESPONDENCE:

Terri V Newman, tvn6@pitt.edu

J Manag Care Spec Pharm 2021;27(4):435-43

Copyright@2021, Academy of Managed Care Pharmacy. All rights reserved.

METHODS: We used claims data from a large pharmacy benefit manager. Patients included were commercially insured adults with type 2 diabetes and a prescription claim for DPP-4i in 2016 or 2017. Using propensity score methods, we matched patients who switched to SGLT2i or GLP-1RA with those who remained on DPP-4i. Among matched samples, we 
conducted multivariable negative binomial regression to examine differences in the incidence of inpatient and emergency room (ER) visits and generalized linear regression to examine differences in health care costs.

RESULTS: Among 47,953 patients who used DPP-4i in 2016 and 2017, 507 switched to SGLT2i and 808 switched to GLP-1RA. Propensity score matching of 1:6 resulted in 3,042 nonswitchers $/ 507$ switchers for the SGLT2i cohort and 4,848 nonswitchers $/ 808$ switchers for the GLP-1RA cohort. Switchers to SGLT2i experienced a 39\% reduction (incidence rate ratio $[\mathrm{IRR}]=0.61,95 \% \mathrm{Cl}=0.38-0.96$ ), and GLP-1RA switchers experienced a $29 \%$ reduction ( $\mathrm{IRR}=0.71,95 \% \mathrm{Cl}=0.52-0.97)$ in inpatient hospitalizations. ER visit rates did not differ significantly between switchers and nonswitchers. Switchers to SGLT2i did not have statistically significant differences in medical or pharmacy costs compared with DPP-4i users, while switchers to GLP-1RA had significantly higher total pharmacy costs (adjusted difference of $\$ 2,453.10$, $95 \% \mathrm{Cl}=\$ 1,837.20-\$ 3,069.00)$.

CONCLUSIONS: Switching from DPP-4i to GLP-1RA or SGLT2i was associated with fewer hospitalizations; however, higher pharmacy costs may outweigh savings from reduced hospitalizations, especially for GLP-1RAs. As newer diabetes guidelines steer specific populations to these drug classes, it is important to optimize drug pricing to realize their true value.

Diabetes is a leading cause of death and disability in the United States, affecting 1 in 10 Americans. ${ }^{1,2}$ In the United States, diabetes is the most expensive health condition, ${ }^{3,4}$ with spending exceeding all other disease areas, including oncology and autoimmune disorders. ${ }^{5}$ Although management of individuals with type 2 diabetes is focused on preventing and delaying the development of long-term complications, until recently, there were few therapeutic options proven to meet those goals. ${ }^{6,7}$

Most individuals with type 2 diabetes require secondline medications in addition to metformin to achieve targeted glycemic levels. ${ }^{6,7}$ While many second-line treatment options exist, the 2018 American Diabetes Association (ADA) guidelines recommend specific glucagon-like peptide-1 receptor agonists (GLP-1RA) and sodium-glucose cotransporter 2 inhibitors (SGLT2i) as second-line agents for those with cardiovascular disease because of evidence that these medications reduce cardiovascular events and mortality..$^{8-10}$ The 2020 ADA guidelines further expand this recommendation to consider use of these newer agents in individuals with established kidney disease or heart failure, regardless of hemoglobin A1c (A1c) levels. ${ }^{11}$

In addition, a 2019 consensus statement from the American Association of Clinical Endocrinologists/ American College of Endocrinology recommends GLP-1RA over SGLT2i and SGLT2i over dipeptidyl peptidase-4 inhibitors (DPP-4i) as second-line therapy for patients with diabetes, regardless of cardiovascular disease status. ${ }^{12}$ Despite guidelines, DPP-4i are nevertheless one of the most commonly used second-line agents, with utilization rates exceeding that of GLP-1RA and SGLT2i. ${ }^{13}$ However, unlike SGLT2i and GLP-1RA, DPP-4i lack evidence for cardiovascular event and mortality reduction. Given the superior clinical outcomes among SGLT2i and GLP-1RA users and the latest society guideline recommendations, clinicians may increasingly switch patients from DPP-4i to SGLT2i and GLP-1RA agents. These newer classes are more costly than DPP-4i, averaging between $\$ 65$ and $\$ 388$ more a month. ${ }^{8,14,15}$ Thus, it is unclear how switching between these medication classes will affect health care utilization and costs. We therefore sought to determine the effect of switching from DPP-4i to GLP-1RA or SGLT2i on health care utilization, medical costs, pharmacy costs, and total costs of care using real-world data.

\section{Methods}

\section{DATA SOURCE AND STUDY POPULATION}

A retrospective cohort study was conducted using medical and pharmaceutical administrative claims data from a large pharmacy benefit manager for the period of January 1 , 2016-December 31, 2018. The medical claims data were provided by a subset of commercial clients receiving pharmacy benefits who did not have any data use restrictions in place. Our study population included commercially insured adults aged 18 years or older, with $\geq 1$ prescription claim for a DPP-4i (sitagliptin, saxagliptin, linagliptin, or alogliptin) in the second quarter of 2016 or 2017 (index period) and an International Classification of Diseases, Tenth Revision, Clinical Modification diagnosis code for type 2 diabetes (ICD-10-CM: E11x) in the 90 days before the index DPP-4i claim (Supplementary Figure 1, available in online article). Patients included in the study were continuously enrolled for 24 months.

We excluded patients who (a) had concomitant use of DPP-4i and SGLT2i or DPP-4i and GLP-1RA, (b) ever had claims for a SGLT2i and GLP-1RA during the study period, and (c) discontinued DPP-4i, SGLT2i, and GLP-1RA in the follow-up period. Discontinuation was defined as no subsequent prescription fill in more than 120 days from the date when the previous fill was exhausted. Data used for this study were deidentified and in full compliance with the Health Insurance Portability and Accountability Act (HIPAA); therefore, an institutional review board approval was not required. 


\section{TABLE 1}

Baseline Characteristics for Matched Cohorts of Nonswitchers and Switchers to SGLT2i and GLP-1RA

\begin{tabular}{|c|c|c|c|c|c|}
\hline \multirow[b]{3}{*}{ Characteristics } & \multirow[b]{3}{*}{ Groups } & \multicolumn{2}{|c|}{ SGLT2i Cohort } & \multicolumn{2}{|c|}{ GLP-1RA Cohort } \\
\hline & & $\begin{array}{c}\text { Nonswitchers } \\
(\mathrm{n}=3,042)\end{array}$ & $\begin{array}{c}\text { Switchers } \\
(n=507)\end{array}$ & $\begin{array}{c}\text { Nonswitchers } \\
(n=4,848)\end{array}$ & $\begin{array}{l}\text { Switchers } \\
(n=808)\end{array}$ \\
\hline & & \multicolumn{2}{|c|}{$\%$ or Mean } & \multicolumn{2}{|c|}{$\%$ or Mean } \\
\hline \multirow[t]{3}{*}{ Age group } & $18-39$ & 9.9 & 9.5 & 9.1 & 10.0 \\
\hline & $40-64$ & 75.7 & 76.1 & 74.4 & 73.5 \\
\hline & $65+$ & 14.4 & 14.4 & 16.5 & 16.5 \\
\hline \multirow[t]{2}{*}{ Gender } & Men & 60.2 & 60.5 & 48.1 & 49.1 \\
\hline & Women & 39.8 & 39.5 & 51.9 & 50.9 \\
\hline \multicolumn{2}{|c|}{ Disease burden proxy ${ }^{a}$} & 7.48 & 7.70 & 9.04 & 8.79 \\
\hline \multirow{2}{*}{$\begin{array}{l}\text { Median ZIP } \\
\text { code-level income }\end{array}$} & $<\$ 48,000$ & 44.1 & 44.4 & 44.1 & 43.1 \\
\hline & $\geq \$ 48,000$ & 55.9 & 55.6 & 55.9 & 56.9 \\
\hline \multirow[t]{2}{*}{$M A^{b}$} & No & 14.4 & 15.0 & 15.9 & 15.6 \\
\hline & Yes & 85.6 & 85.0 & 84.1 & 84.4 \\
\hline HF status $^{c}$ & With $\mathrm{CHF}$ & 2.7 & 1.8 & 4.0 & 2.9 \\
\hline Other CVD status ${ }^{d}$ & With other CVD & 13.8 & 13.4 & 16.1 & 14.4 \\
\hline $\begin{array}{l}\text { Previous diabetes } \\
\text { drug use }\end{array}$ & $\begin{array}{l}\text { 30-day adjusted } \\
\text { prescriptions }\end{array}$ & 4.89 & 4.98 & 5.15 & 5.61 \\
\hline Infection history & $\begin{array}{l}\text { Yeast infection or } \\
\text { UTI history }\end{array}$ & 6.0 & 5.9 & - & - \\
\hline
\end{tabular}

Note: None of the differences in covariates were statistically significant between nonswitchers and switchers in the SGLT2i and GLP-1RA cohorts.

a Measure that reflects the number of distinct drug groups used by patients based on unique 2-digit Generic Product Identifier codes, except for diabetes.

${ }^{b}$ Defined as metropolitan or micropolitan statistical area.

'Patients with >1 medical claim with a diagnosis for heart failure (see Supplementary Table 3 for diagnoses codes).

dPatients with >1 medical claim with a diagnosis for other cardiovascular diseases (see Supplementary Table 3 for diagnoses codes).

eNumber of 30-day adjusted prescriptions for diabetes drugs in the preperiod.

'Patients with $>1$ medical claim with a diagnosis for yeast infection and/or UTI infection. Not included in the GLP-IRA model.

$\mathrm{CHF}=$ congestive heart failure; $C V D=$ cardiovascular disease; $G L P-1 R A=$ glucagon-like peptide-1 receptor agonist; $H F=$ heart failure; $M S A=$ metropolitan statistical area; $S G L T 2 i=$ sodium-glucose cotransporter 2 inhibitor; $\mathrm{UTI}=$ urinary tract infection.

\section{STUDY DESIGN}

To evaluate cost and utilization outcomes for DPP-4i, SGLT2i, and GLP-1RA users, we developed 2 cohorts (cohort 1: January 2016-December 2017; cohort 2: January 2017-December 2018). Within each cohort, we developed 2 sets of comparisons. The first set compared SGLT2i switchers with those who continued using DPP- $4 \mathrm{i}$, and the second set compared GLP-1RA switchers with continuing DPP-4i users.

Switchers were defined as patients who had greater than 120 continuous days of supply for SGLT2i or GLP-1RA, without a gap in therapy, which began after the last day of the DPP-4i fill. For each cohort we used 1 calendar year, defined as the "switch assessment year," to evaluate baseline DPP-4i use and switching patterns (cohort 1:
January 1-December 31, 2016; cohort 2: January 1-December 31, 2017). We then used the following consecutive year, defined as the "outcomes assessment year," to observe cost and utilization outcomes (cohort 1: January 1-December 31, 2017; cohort 2: January 1-December 31, 2018).

Supplementary Figure 1 (available in online article) shows the switch assessment year and outcomes assessment year in more detail. The switch assessment year was used to identify patients with type 2 diabetes who were prevalent users of DPP- $4 \mathrm{i}$ and who switched to a SGLT2i or GLP1RA. The switch assessment year was divided into 3 segments: preperiod, index period, and switch evaluation period. The preperiod was used to identify individuals with a diagnosis of type 2 diabetes, determine new or continuous users of DPP- 4 i, and evaluate previous use of SGLT2i or GLP-1RA within the first quarter of the year. The index period was used to identify patients with any prescription claim for a DPP-4i within the second quarter of the year. In the switch evaluation period, we evaluated whether DPP-4i users switched to GLP-1RA or SGLT2i within the last 180 days of the year.

Patients were classified as switchers if they switched to a SGLT2i or a GLP-1RA and continued using the switched drug class throughout the remainder of the study period. We defined our nonswitcher comparison group as all patients who remained on a DPP-4i for the remaining duration of the study. Finally, we assessed our outcomes of interest by examining differences in health care utilization and health care costs between switchers and nonswitchers in the outcomes assessment year.

\section{OUTCOMES}

Our study included 2 primary outcomes: health care utilization and costs. Health care utilization was 


\section{FIGURE 1 Incidence Rate Ratio of Health Care Utilization for Switchers to SGLT2i Compared with Prevalent DPP-4i Users (Nonswitchers)}

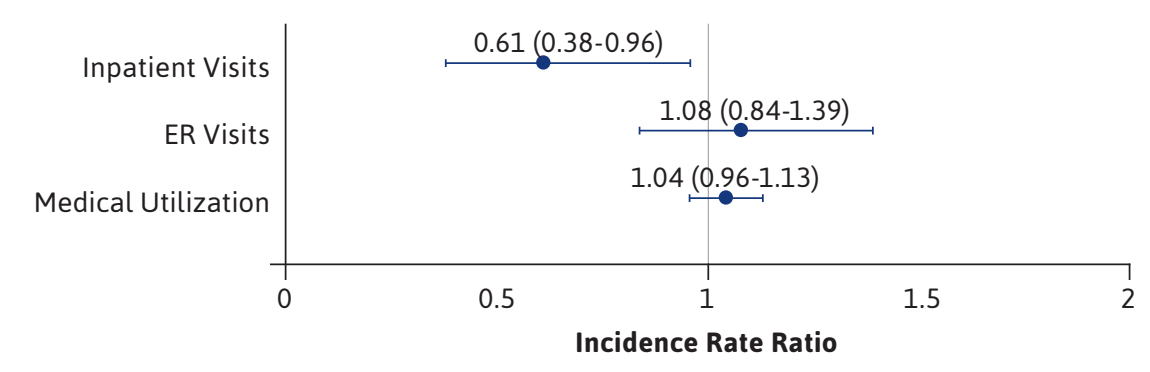

Notes: This figure shows the adjusted IRR and $95 \%$ confidence intervals of inpatient and ER visits in the outcome assessment year for switchers to SGLT2i compared with those who remained on DPP-4i in the switch assessment year. Analyses were conducted using negative binomial regression models adjusted for age, gender, disease burden proxy, income, urban/rural residence, history of cardiovascular disease, urinary tract infection, and yeast infection history.

$D P P-4 i=$ dipeptidyl peptidase -4 inhibitor; $E R=$ emergency room; $I R R=$ incidence rate ratio; $S G L T 2 i=$ sodiumglucose cotransporter 2 inhibitor.

defined as the rate of all-cause inpatient visits, emergency room (ER) visits, and total medical utilization (sum of all hospitalizations, outpatient and physician office visits, diagnostics, and other medical procedures and services) between DPP-4i switchers and nonswitchers. Healthcare costs were defined as annual per-patient total prescription drug plan costs (net of rebates, includes ingredient costs, taxes, dispensing fees, and administrative fees); all-cause inpatient, ER visit, and total medical costs (paid amount); and total health care costs (sum of medical and drug plan costs).

\section{COVARIATES}

We adjusted for baseline characteristics that included age (as of January 1 of the switch assessment year); gender; median family income proxy at the residence ZIP code level (obtained from 2010 U.S. Census data); disease burden proxy (a measure that reflects the number of distinct drug groups used by patients based on unique 2-digit Generic Product Identifier codes, except for diabetes); urban or rural area of residence (defined as metropolitan or micropolitan statistical area); number of 30-day adjusted prescriptions for diabetes in the preperiod; and diagnosis of congestive heart failure and other cardiovascular disease conditions (see Supplementary Table 3 for diagnoses codes, available in online article).

For medical utilization cost outcomes, we adjusted for total preperiod medical utilization and costs in the multivariable analysis. For the model that compared switchers to SGLT2i with nonswitchers, we additionally adjusted for history of urinary tract infection or yeast infection diagnoses, since SGLT2i use has been associated with higher urinary tract infection and yeast infection rates (see Supplementary Table 3 for diagnoses codes, available in online article). ${ }^{16}$

\section{STATISTICAL ANALYSIS}

We conducted statistical analyses between each pair of switcher groups (GLP-1RA or SGLT2i) and the corresponding comparator group of prevalent DPP-4i users. To compare baseline patient characteristics between each switcher group and its corresponding comparator group of prevalent DPP-4i users, we used chi-square tests and 2-sample t-tests accordingly. We then conducted a 1:6 propensity score matching on the previously mentioned baseline covariates to minimize potential selection bias between switcher and comparator groups. We used multivariable negative binomial regression to examine differences in incidence of inpatient and ER visits, and generalized linear regression, with identity link and normal distribution, to examine health care costs. All analyses were conducted using SAS 9.4 (SAS Institute, Cary, NC).

To determine if there were any changes in directionality or statistical significance for utilization and cost outcomes before matching, we conducted a sensitivity analysis by replicating the main analyses in the unmatched sample. Also, since SGLT2i and GLP-1RA have demonstrated reduced cardiovascular-related morbidity and mortality compared with DPP-4i, we conducted subgroup analyses by limiting our sample to individuals diagnosed with cardiovascular disease and re-ran our matched cohort regression models without the cardiovascular disease diagnosis covariate.

\section{Results}

\section{PATIENT CHARACTERISTICS}

We identified 47,953 patients who were using DPP-4i during the index periods in 2016 and 2017. Among these patients, 507 switched to SGLT2i; 808 switched to GLP-1RA; and 46,638 remained on DPP-4i (nonswitchers). The 1:6 propensity score matching resulted in the selection of 3,042 nonswitchers and 507 switchers for the SGLT2i cohort and 4,848 nonswitchers and 
FIGURE 2 Incidence Rate Ratio of Health Care Utilization for Switchers to GLP-1RA Compared with Prevalent DPP-4i Users (Nonswitchers)

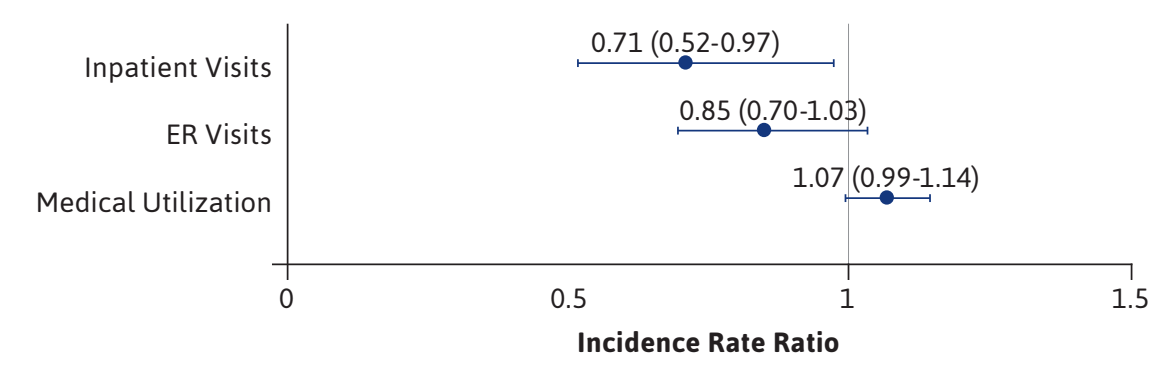

Notes: This figure shows the adjusted IRRs and 95\% confidence intervals of inpatient and ER visits in the outcome assessment year for switchers to GLP-IRA compared with those who remained on DPP-4i in the switch assessment year. Analyses were conducted using negative binomial regression models adjusted for age, gender, disease burden proxy, income, urban/rural residence, and history of cardiovascular disease. DPP- $4 i=$ dipeptidyl peptidase -4 inhibitor; $E R=$ emergency room; $I R R=$ incidence rate ratio; $S G L T 2 i=$ sodium glucose cotransporter 2 inhibitor.

808 switchers for the GLP-1RA cohort. The matched cohorts were comparable with respect to only the covariates previously described. Table 1 shows baseline characteristics for the propensity-matched cohort of SGLT2i and GLP-1RA users respectively.

\section{HEALTH CARE UTILIZATION}

Supplementary Table 1 (available in online article) shows the unadjusted analyses of all outcomes among switchers to SGLT2i and GLP-1RA. Switchers to SGLT2i and GLP-1RA had lower inpatient visits on average compared with patients who remained on DPP-4i (0.05 vs. 0.08, $P=0.043$, and 0.07 vs. $0.13, P=0.003)$. Only the GLP-1RA switchers had lower ER visits compared with their nonswitcher counterparts ( 0.19 vs. $0.24, P=0.023)$. Total medical utilization differences were not statistically significant. Figures 1 and 2 show the adjusted incidence rate ratios (IRR) for inpatient and ER visits and total medical utilization for switchers to SGLT2i and GLP1RA compared with prevalent DPP-4i users, respectively. Switchers to SGLT2i had a 39\% lower incidence of inpatient visits compared with DPP-4i users $(\mathrm{IRR}=0.61,95 \% \mathrm{CI}=0.38-0.96)$.

No statistically significant differences in ER visits (IRR=1.08, 95\% $\mathrm{CI}=0.84-1.39)$ or total medical utilization $\quad(\mathrm{IRR}=1.04,95 \% \mathrm{CI}=0.96-1.13)$ were found. In exploratory analyses, we found that chest and precordial pain were leading causes of ER visits among SGLT2i switchers. Results from Figure 2 show that switchers to GLP1RA had a 29\% lower incidence of inpatient visits compared with DPP-4i users (IRR $=0.71,95 \% \mathrm{C}=0.52-0.97)$ and no significant differences in ER visits $(\mathrm{IRR}=0.85,95 \% \mathrm{CI}=0.70-1.03)$ or total medical utilization $(\mathrm{IRR}=1.07,95 \%$ $\mathrm{CI}=0.99-1.14)$.

\section{HEALTH CARE COSTS}

In the unadjusted analyses, switchers to GLP-1RA had lower mean total medical costs per patient $(\$ 6,091.30$ vs. $\$ 8,348.80, \quad P=0.03) \quad$ compared with nonswitchers (Supplementary Table 1, available in online article). On the other hand, switchers to SGLT2i and GLP-1RA had higher mean total pharmacy costs per patient compared with their respective nonswitcher counterparts $(\$ 5,239.90$ vs. $\$ 4,136.40$, $\mathrm{P}=0.042$ and $\$ 7,153.30$ vs. $\$ 4,811.20$, respectively; $\mathrm{P}<0.001)$. Figures 3 and 4 show the multivariable analysis results for differences in health care costs between switchers to SGLT2i and GLP-1RA, respectively, as compared with prevalent DPP-4i users. Switchers to SGLT2i did not have statistically significant differences in inpatient, ER, total medical, pharmacy, or total health care costs (Figure 3). On the other hand, switchers to GLP1RA had significantly higher pharmacy costs (adjusted difference of $\$ 2,453.10$; $95 \%$ CI $=\$ 1,837.20-\$ 3,069.00)$ with no statistically significant difference in inpatient, ER, and total medical and health care costs (Figure 4).

\section{SUBGROUP AND SENSITIVITY ANALYSES}

In subgroup analyses, we restricted the sample to those diagnosed with cardiovascular disease. Among those with cardiovascular disease, 68 patients switched to SGLT2i, while 419 patients remained on DPP-4i. Additionally, 116 patients switched to GLP-1RA, whereas 779 patients remained on DPP-4i. Switchers to SGLT2i in this subgroup had no differences in inpatient visits (IRR $=0.56$, $95 \% \mathrm{CI}=0.27-1.08)$; $\mathrm{ER}$ visits (IRR $=1.37$, 95\% CI $=0.87-2.14)$; and total medical utilization $(\mathrm{IRR}=0.90,95 \% \mathrm{CI}=0.76$ 1.07) compared with DPP-4i users (Supplementary Figure 2, available in online article). On the other hand, switchers to GLP-1RA had significantly lower inpatient visits (IRR $=0.60,95 \%$ $\mathrm{CI}=0.37-0.96)$, but no difference in ER visits $(\mathrm{IRR}=0.67,95 \% \mathrm{CI}=0.44-1.01)$ and medical utilization (IRR $=1.04,95 \%$ $\mathrm{CI}=0.91-1.19)$, compared with DPP-4i users (Supplementary Figure 3, available in online article).

When examining health care costs among patients with cardiovascular disease, switchers to SGLT2i did not have statistically significant 


\section{FIGURE 3 Health Care Costs for Switchers to SGLT2i Compared with Prevalent DPP-4i Users (Nonswitchers)}

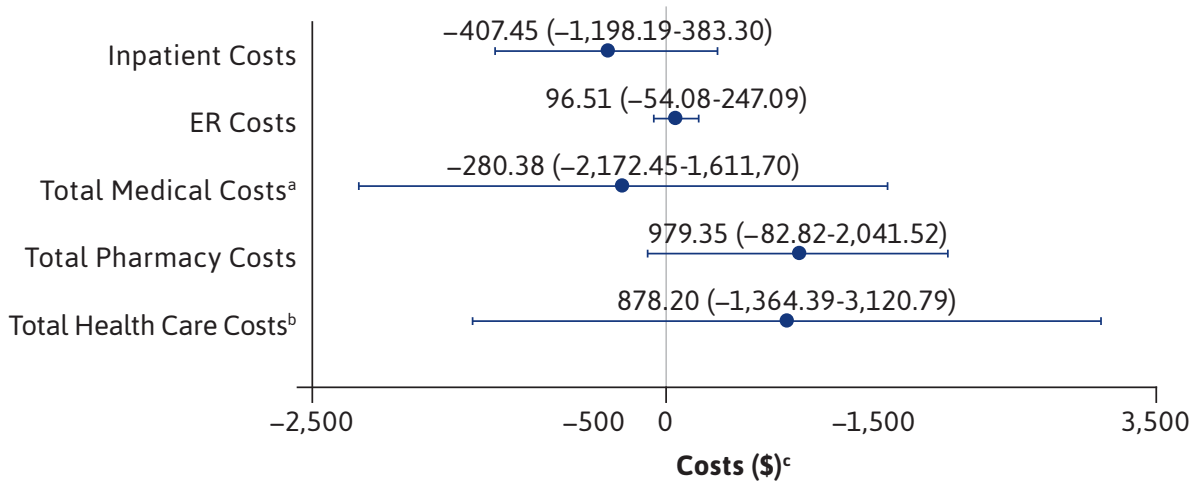

Notes: This figure shows the difference in inpatient, ER, total medical, total pharmacy, and total health care costs in the outcome assessment year for switchers to SGLT2i as compared with those who did not switch and remained on DPP- $4 i$ in the switch assessment year. Analyses were conducted using negative binomial regression models adjusted for age, gender, disease burden proxy, income, urban/rural residence, history of cardiovascular disease, urinary tract infection, and yeast infection history.

aTotal medical costs were defined as all nondrug services.

${ }^{b} T o t a l$ health care costs were evaluated as the sum of pharmacy and medical costs.

'Costs were reported as adjusted costs with $95 \%$ confidence intervals.

$D P P-4 i=$ dipeptidyl peptidase 4 inhibitor; $E R=$ emergency room; $I R R=$ incidence rate ratio; $S G L T 2 i=$ sodium glucose cotransporter 2 inhibitor.

differences in costs compared with DPP-4i users, similar to the main analysis (Supplementary Figure 4, available in online article). On the other hand, switchers to GLP-1RA had significantly higher pharmacy costs (adjusted difference of $\$ 2,443.50,95 \%$ $\mathrm{CI}=\$ 1,827.28-\$ 3,059.71) \quad$ compared with prevalent DPP-4i users with no statistically significant difference in inpatient, ER, total medical, and total health care costs (Supplementary Figure 5, available in online article).

Results from our sensitivity analyses using the traditional covariate adjustment method in the unmatched sample led to similar findings as our main analysis.

\section{Discussion}

In this analysis, we examined how health care utilization and costs differ between continuous DPP-4i users and those who switched to SGLT2i or GLP-1RA. Compared with those who remained on DPP- $4 \mathrm{i}$, switchers to SGLT2i or GLP-1RA experienced a $39 \%$ and $29 \%$ reduction in incidence of inpatient hospitalizations, respectively. Switchers to GLP-1RA showed nonsignificant trends towards lower rates of ER visits, whereas switchers to SGLT2i displayed nonsignificant trends towards increased rates of ER visits. Use of all medical services did not differ between the switcher and nonswitcher cohorts.

Regarding costs, switchers to GLP-1RA and SGLT2i demonstrated no differences in total medical and health care costs, whereas switchers to GLP-1RA had significantly higher pharmacy costs. Taken together, our results show that despite reductions in inpatient hospitalizations observed with switching to SGLT2i or GLP-1RA, there were no differences in total costs of care. This could be attributed to the higher pharmacy costs associated with the use of these agents, especially in the case of GLP-1RA.

Restricting the analyses to individuals with cardiovascular disease, we found that switchers to GLP-1RA had lower inpatient visits and higher pharmacy costs. Within this population of individuals with cardiovascular disease, switchers to SGLT2i and GLP-1RA experienced no difference in total health care costs, which was in part because of higher ER costs for SGLT2i switchers and higher pharmacy costs for GLP-1RA switchers.

To our knowledge, this is the first study to directly compare health care utilization and cost outcomes among DPP-4i, GLP-1RA, and SGLT2i users. With the introduction of these newer agents, treatment options for patients have expanded, increasing the need for comparative effectiveness research to compare key outcomes among these agents. Comparative effectiveness literature directly comparing economic and health care utilization outcomes among these 3 novel drugs is limited. A cost-effectiveness analysis of patients inadequately controlled on metformin monotherapy compared SGLT2i with other second-line diabetes treatment regimens, including DPP-4i and GLP-1RA. It found that SGLT2i users experienced lower total medical costs compared with GLP1RA and DPP-4i users. ${ }^{17}$

In contrast, our study found that compared with prevalent DPP-4i users SGLT2i and GLP-1RA switchers experienced no difference in medical costs. This difference could be attributed to our focus on those who switched between drug classes rather than focusing solely on new users. Furthermore, in line with our findings, a study by Li et al. (2014) found that, compared with sitagliptin (a DPP-4i) 


\section{FIGURE 4 Health Care Costs for Switchers to GLP-1RA Compared with Prevalent DPP-4i Users (Nonswitchers)}

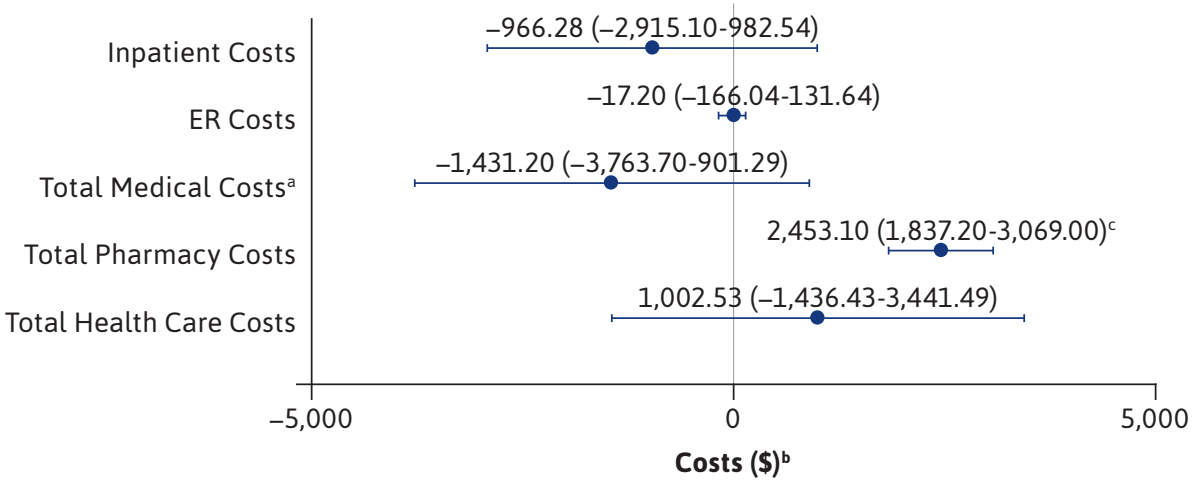

Notes: This figure shows the difference in inpatient, emergency room, total medical, total pharmacy, and total health care costs in the outcomes assessment year for switchers to GLP-IRA as compared with those who did not switch and remained on DPP-4i in the switch assessment year.

${ }^{a}$ Total medical costs were defined as all nondrug services, and total health care costs were evaluated as the sum of pharmacy and medical costs.

${ }^{b}$ Costs were reported as adjusted costs with $95 \%$ confidence intervals. All generalized linear models to assess health care costs among switchers to GLP-1RA versus DPP-4i users were adjusted for age, gender, disease burden proxy, income, urban/rural residence, and history of cardiovascular disease.

${ }^{C} P<0.05$ indicated statistically significant difference in costs between switchers and nonswitchers.

$D P P-4 i=$ dipeptidyl peptidase -4 inhibitor; $E R=$ emergency room; IRR = incidence rate ratio; $S G L T 2 i=$ sodiumglucose cotransporter 2 inhibitor.

users, liraglutide (a GLP-1RA) users had lower diabetes-related medical costs but higher diabetes-related pharmacy costs, resulting in overall similar total cost of care between the 2 drugs. ${ }^{18}$

Our study has important implications. First, despite lower hospitalization rates among switchers to SGLT2i or GLP-1RA compared with nonswitchers, greater pharmacy costs may have mitigated any difference in total costs of care. Consequently, the value of these novel agents in reducing total cost of care for diabetes patients will not be fully realized without addressing drug pricing.

Second, switchers to either a SGLT2i or GLP-1RA experienced fewer inpatient hospitalizations; however, these averted admissions did not translate to reduced inpatient costs. This could imply that SGLT2i and GLP-1RA users represent a sicker population with higher inpatient admissions and related costs. Although we did adjust for comorbidities using a disease burden proxy, we could have underestimated the disease burden in this population.

Finally, in subgroup analyses, we restricted our population to individuals with a cardiovascular disease diagnosis and observed a trend towards lower total costs of care among this population. This differed from the main analysis where switching was associated with a trend towards higher total health care costs. To further examine this difference, we conducted additional analyses to explore how total health care costs compared for individuals with and without cardiovascular disease
(Supplementary Table 2, available in online article).

Our results showed that among individuals without cardiovascular disease, switchers to GLP-1RA and SGLT2i had greater total costs of care. In contrast, among those with cardiovascular disease, switchers to GLP-1RA and SGLT2i experienced no difference in total cost of care with a trend towards lower costs of care among switchers (Supplementary Table 2).

Findings from these subanalyses may reflect the cardiovascular benefit associated with these newer agents in patients with established cardiovascular disease. Further research should compare how utilization and economic outcomes differ with SGLT2i and GLP-1RA between patients with and without cardiovascular disease.

\section{LIMITATIONS}

Our study has some limitations to consider. First, outcomes in this study were observed for a duration of 1 year. Using a longer follow-up time may demonstrate a greater long-term benefit of switching to SGLT2i and GLP-1RA, since clinical studies have indicated ongoing cardiovascular benefit beyond 1 year. ${ }^{19}$

Second, our cohort contained a relatively small percentage of switchers. This could be related to the release of new society guidelines in 2018 and 2019 that recommended the use of SGLT2i and GLP-1RA, while we defined our switching population based on switching trends in 2016 and 2017. ${ }^{8}$ We anticipate that as varying society guidelines are disseminated to practice, switching to SGLT2i and GLP-1RA will become more prevalent and data behind switching will become more robust. 
Third, our analysis was limited to the covariates available in our datasets, thus, limiting our ability in adjusting for race/ethnicity, clinical and lab values, such as A1c, or social determinants of health.

Fourth, it is possible that a primary reason for switching from DPP-4i to SGLT2i and GLP-1RA is related to diabetes progression, thereby resulting in our switching populations having more advanced disease than prevalent DPP-4i users. However, if switchers to SGLT2i and GLP-1RA were sicker than prevalent DPP-4i users, one would expect that they would have higher inpatient admissions and medical costs; it is therefore particularly notable that this trend was not observed among switchers.

Finally, we limited our study to members with continuous insurance eligibility in order to estimate the average treatment effect on those treated with SGLT2i or GLP-1RA compared with DPP-4i. As such, our findings may not be generalizable to those without continuous insurance eligibility.

\section{Conclusions}

Although switching from a DPP-4i to GLP-1RA and SGLT2i may result in fewer inpatient hospitalizations, higher pharmacy costs may outweigh medical savings associated with reduced inpatient utilization, especially for GLP-1RA. The uptake of these agents are expected to increase as newer diabetes guidelines broaden the use of these drug classes in specific populations (individuals with cardiovascular disease, chronic kidney disease, heart failure, or other high-risk factors) regardless of A1c levels. Therefore, optimization of drug pricing is important in order to realize the true value of these innovative treatments over time.

\section{DISCLOSURES}

No outside funding supported this study. Neilson, Good, Swart, and Huang are employees of UPMC Center for ValueBased Pharmacy Initiatives and High-Value Care. Parekh reports employment at UPMC until July 2019. Munshi and Henderson are employed by Express Scripts. Newman has no disclosures to report.

\section{REFERENCES}

1. National Center for Chronic Disease Prevention and Health Promotion. About chronic diseases. 2019. Accessed February 24, 2021. https://www.cdc.gov/chronicdisease/about/index.htm

2. Centers for Disease Control and Prevention. Type 2 diabetes. 2019. Accessed February 24, 2021. https://www. cdc.gov/diabetes/basics/type2.html

3. Cefalu W. Diabetes: our nation's most expensive health condition. U.S. News and World Report. March 28, 2018. Accessed February 24, 2021. https://health. usnews.com/health-care/for-better/ articles/2018-03-28/diabetes-ournations-most-expensive-health-condition

4. American Diabetes Association.

Economic costs of diabetes in the U.S. in 2017. Diabetes Care. 2018;41(5):917-28.

5. QuintilesIMS Institute. Medicines use and spending in the U.S. A review of 2016 and Outlook to 2021. May 2017. Accessed February 24, 2021. https://structurecmsstaging-psyclone.netdna-ssl.com/ client assets/dwonk/media/attachments /590c/6aa0/6970/2d2d/4182/0000/590 c6aa069702d2d41820000.pdf?1493985952

6. American Diabetes Association.

Standards of Medical Care in

Diabetes-2019 Abridged for Primary Care Providers. Clin Diabetes. 2019;37(1):11-34.

7. Zhang Y, McCoy RG, Mason JE, Smith SA, Shah ND, Denton BT. Second-line agents for glycemic control for type 2 diabetes: are newer agents better? Diabetes Care. 2014;37(5):1338-45.

8. American Diabetes Association. 8. Pharmacologic approaches to glycemic treatment: Standards of Medical Care in Diabetes-2018. Diabetes Care. 2018;41(Suppl 1):S73-S85.
9. Zheng SL, Roddick AJ, Aghar-Jaffar R, et al. Association between use of sodiumglucose cotransporter 2 inhibitors, glucagon-like peptide 1 agonists, and dipeptidyl peptidase 4 inhibitors with allcause mortality in patients with type 2 diabetes: a systematic review and metaanalysis. JAMA. 2018;319(15):1580-91.

10. Zelniker TA, Wiviott SD, Raz I, et al. Comparison of the effects of glucagon-like peptide receptor agonists and sodiumglucose cotransporter 2 inhibitors for prevention of major adverse cardiovascular and renal outcomes in type 2 diabetes mellitus. Circulation. 2019;139(17):2022-31.

11. American Diabetes Association. 9. Pharmacologic approaches to glycemic treatment: Standards of Medical Ccare in Diabetes-2020. Diabetes Care. 2020;43(Suppl 1):S98-S110.

12. Garber AJ, Abrahamson MJ, Barzilay JI, et al. Consensus statement by the American Association of Clinical Endocrinologists and American College of Endocrinology on the comprehensive type 2 diabetes management algorithm-2019 executive summary. Endocr Pract. 2019;25(1):69-100.

13. Montvida O, Shaw J, Atherton JJ, Stringer F, Paul SK. Long-term trends in antidiabetes drug usage in the U.S.: real-world evidence in patients newly diagnosed with type 2 diabetes. Diabetes Care. 2018;41(1):69-78.

14. Edwards KL, Minze MG. Dulaglutide: an evidence-based review of its potential in the treatment of type 2 diabetes. Core Evid. 2015;10:11-21.

15. IBM. Micromedex Red Book Online. Database. Accessed February 24, 2021. https://www.ibm.com/products/ micromedex-red-book

16. Li D, Wang T, Shen S, Fang Z, Dong Y, Tang H. Urinary tract and genital infections in patients with type 2 diabetes treated with sodium-glucose co-transporter 2 inhibitors: a meta-analysis of randomized controlled trials. Diabetes Obes Metab. 2017;19(3):348-355. 
17. Chakravarty A, Rastogi M, Dhankhar P, Bell KF. Comparison of costs and outcomes of dapagliflozin with other glucose-lowering therapy classes added to metformin using a short-term costeffectiveness model in the U.S. setting. J Med Econ. 2018;21(5):497-509.
18. Li Q, Chitnis A, Hammer M, Langer J. Real-world clinical and economic outcomes of liraglutide versus sitagliptin in patients with type 2 diabetes mellitus in the United States. Diabetes Ther. 2014;5(2):579-90.
19. Røder ME. Major adverse cardiovascular event reduction with GLP-1 and SGLT2 agents: evidence and clinical potential. Ther Adv Chronic Dis. 2018;9(1):33-50. 